\title{
The copper-beaten skull
}

\section{N Mahomed, MB BCh, FCRad (D) \\ T Sewchuran, $M B B C h$ \\ Z Mahomed, $M B B C h$}

Department of Radiology, University of the Witwatersrand, Johannesburg

Corresponding author: N Mahomed (nasreen.mahomed@wits.ac.za)

\begin{abstract}
Variations and abnormalities of skull appearance and shape are generally related to a primary maldevelopment of the brain. The copper-beaten skull appearance is typically associated with craniosynostosis, where premature fusion of the cranial bone sutures results in the growing brain exerting pressure on the malleable cranium, producing a pattern known as the copper-beaten skull appearance.
\end{abstract}

A 6-year-old girl with known craniosynostosis graphically demonstrates the copper-beaten skull on plain radiography (Fig. 1).

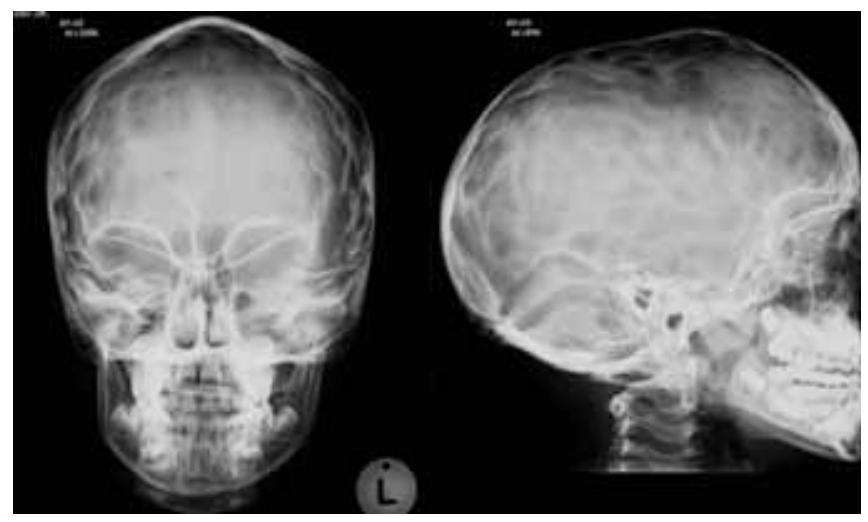

Fig. 1. PA and lateral skull $X$-rays demonstrating impressive convolutional markings (copper-beaten skull appearance) in a 6-year-old girl with known craniosynostosis. Note associated fusion of the saggital, coronal and lamdoid sutures.

Variations and abnormalities of skull appearance and shape are often related to a primary maldevelopment of the brain. ${ }^{1}$ The copper-beaten skull appearance is typically associated with craniosynostosis, which is the premature fusion of the cranial bone sutures (Fig. 2). ${ }^{2}$ Severe craniosynostosis and reduced cranial growth in our patient caused brain volume to exceed the cranium size, resulting in raised intracranial pressure. ${ }^{2}$ Consequently, the growing brain exerts a continuous pulsatile pressure on the malleable cranium, producing a gyral pattern evidenced on plain skull X-rays most commonly known as the copper-beaten skull appearance. ${ }^{3}$ This is the least common manifestation of craniosynostosis on the developing skull.

Convolutional markings are inner table indentations that conform to the cerebral surface of the growing brain in infants. ${ }^{1}$ The pattern was initially assumed to have resulted from chronically elevated intracranial pressure but currently it is widely considered to be a reflection of normal brain growth, without pathological significance. ${ }^{4}$ The markings are most prominent during periods of rapid brain growth, between age $2-3$

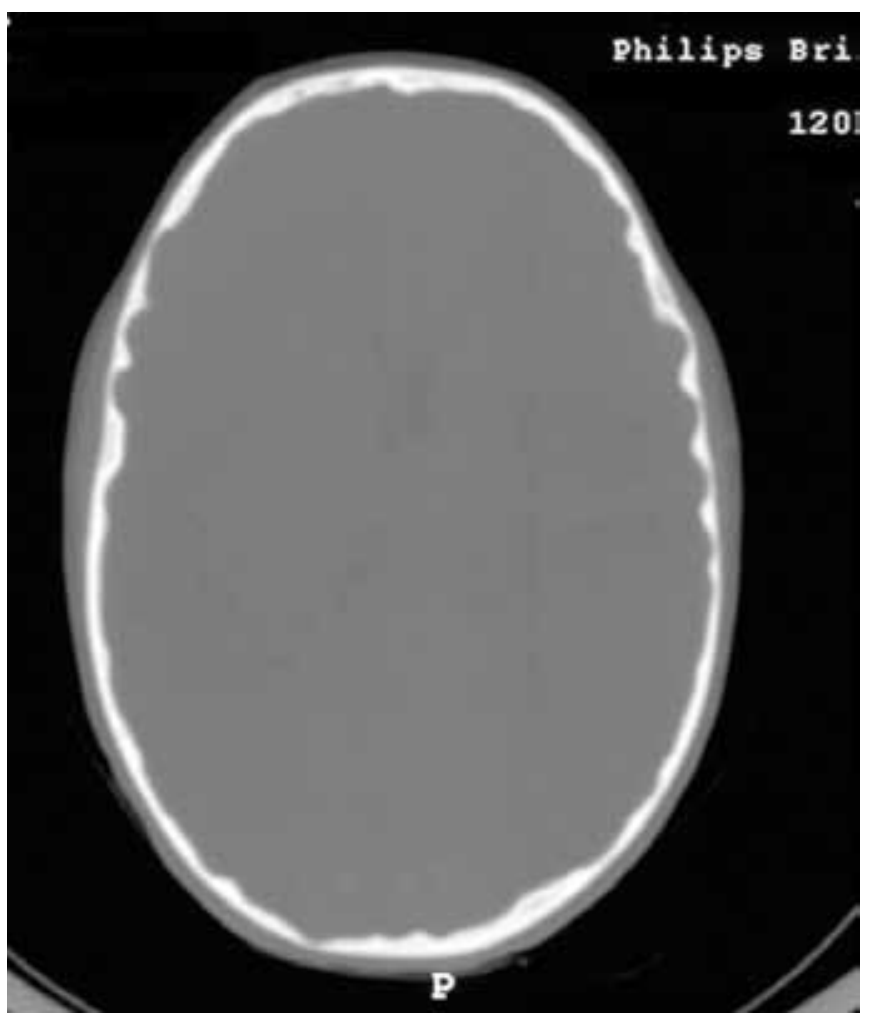

Fig. 2. Axial CT of brain bony windows, demonstrating the convolutional markings of the inner table of the skull that conform to the cerebral surface of the growing brain associated with fusion of the coronal and lamdoid sutures.

years and 5 - 7 years. They become less prominent after approximately 8 years of age. ${ }^{1}$

Diffuse, severe beaten-copper pattern is an indicator of chronic elevated intracranial pressure, which is more common in patients with craniosynostosis. ${ }^{4}$ Additional findings associated with a chronic increase in intracranial pressure include macrocrania, splitting of the sutures, skull demineralisation and erosion, or enlargement of the sella turcica. ${ }^{1}$ The appearance is more frequent in children with complex, rather than simple, craniosynostosis. ${ }^{2}$ In children $<18$ months, the presence of a diffuse copper-beaten pattern on skull radiography, together with narrowing of the basal cisterns and obliteration of the anterior sulci, increases the likelihood of raised intracranial pressure. ${ }^{2,4}$

Of interest, Van Der Meulen et al. concluded that the presence of the copper-beaten skull pattern did not significantly affect long-term intelligence levels. ${ }^{2}$ However, there appeared to be a negative influence on more subtle areas of development, such as reading, spelling and behaviour. $^{2}$ 


\section{PICTORIAL INTERLUDE}

These convolutional markings should be differentiated from Lückenschädel (lacunar skull), which is due to mesenchymal dysplasia of calvarial ossification. It is characterised by numerous oval lucencies

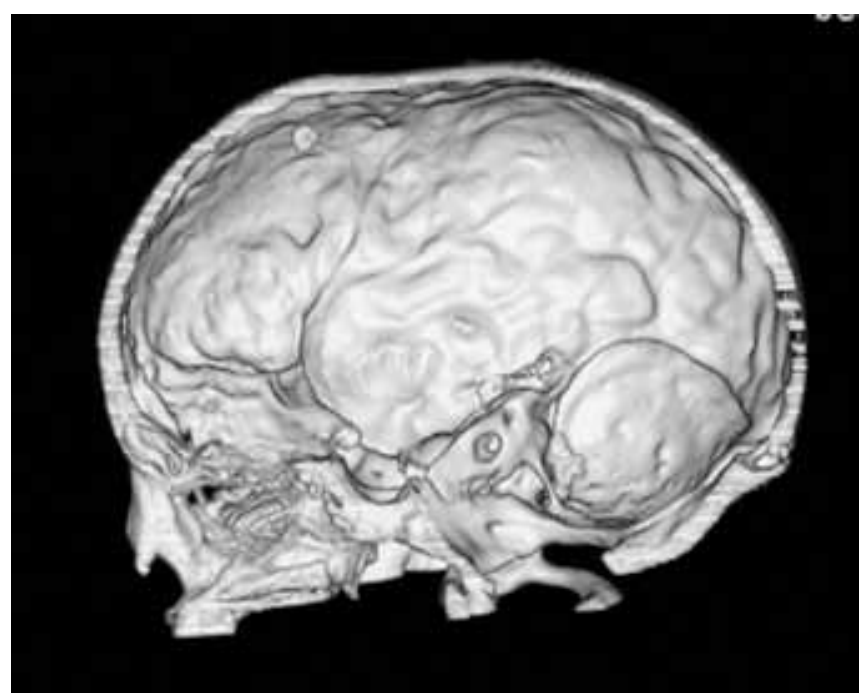

Fig. 3. CT 3D saggital reconstruction demonstrating the prominent convolutional markings of the inner table of the skull. There is no obvious expansion or erosion of the sellar turcica. (a honeycomb appearance about $2 \mathrm{~cm}$ in diameter) of the inner table and diploic space. ${ }^{1,4}$ These are usually present at birth and are associated with meningocoele/myelomeningocoele/encephalocoele, spina bifida, cleft palate and Arnold Chiari II malformation. ${ }^{1}$ It is most prominent in the parietal and occipital bones. Occasionally these findings are normal and disappear by 6 months of age. ${ }^{1}$

Craniosynostosis is more adequately evaluated for surgical planning with computer tomography (CT) and 3D reconstructions (Fig. 3), and has the advantage of concurrently demonstrating the intracranial complications. The associated interpretation of plain-film skull radiography is an incidental contribution.

Acknowledgements. We thank Professor S. Andronikou for reviewing this article.

1. Glass BJR, Fernbach SK, Norton KI, Choi SP, Naidich TP. The infant skull : A vault of information. Radiographics 2004;24:507-522.

2. Van der Meulen J, Van der Vlugt J, Okkerse J, Hoffman B. Early beaten-copper pattern: its long term effect on intelligence quotients in 95 children with craniosynostosis. J Neurosurgery: Paediatrics 2008;1:25-30.

3. Agrawal D, Steinbok P, Cochrane DD. Significance of beaten copper appearance on skull radiographs in children with isolated sagittal synostosis. Childs Nerv Syst 2007;23:1467-1470.

4. El Khasab M, Nejat F, Yazdani s, Baradaran N. Aquired craniomeningocele in an infant with craniosynostosis : a case report. Journal of Medical Case Reports 2010;4:104-106. 